\title{
Deriving Fundamental Solutions for Equations of Elastodynamics in Three-Dimensional Cubic Quasicrystals
}

\author{
H. ÇERDik Yaslan*
}

Department of Mathematics, Pamukkale University, Denizli, 20070, Turkey

(Received April 25, 2019; revised version May 10, 2019; in final form July 07, 2019)

Cubic quasicrystal (QC) is one of the important three-dimensional QCs. In this paper, according to Bak's arguments, dynamic elasticity equations for cubic QCs are considered. Fundamental solutions (FSs) of the phonon-phason displacements, displacement speeds, and stresses arising from pulse point sources are computed.

DOI: 10.12693/APhysPolA.136.474

PACS/topics: anisotropic dynamic elasticity, cubic quasicrystals, three dimensional quasicrystals, fundamental solution

\section{Introduction}

The three-dimensional QCs include icosahedral QCs and cubic QCs. Cubic QC has a 3D structure quasiperiodic in three orthogonal directions that supports simultaneously phonon and phason fields [1]. Feng et al. [2-4] have reported cubic QCs with cubic symmetry and Wang et al. [5] have discussed the projection description of the cubic QCs. Yang et al. [6] have studied the linear elasticity theory. There are still many physical properties of the cubic quasicrystals which have not been studied yet.

In the literature, there is not much work on solutions of the cubic QCs. For elasticity problems of cubic QCs, a large number of analytical results have been obtained for static cases. In [7-9], plane problems with simpler structure of the cubic QCs have been studied for static case. Based on the complex potential method, plane problems of cubic QC media containing an elliptic hole subjected to uniform remote loadings have been solved in [7]. Equations of plane elasticity of cubic QCs have been simplified to an eighth-order partial differential governing equation and general solutions have been established by using an operator method [8]. The problem of an infinite plane which is composed of two halfplanes with different cubic QC has been investigated in [9]. A method for analyzing the static elasticity problem of cubic QC has been given and the solutions of elastic field of cubic QC with a penny-shaped crack have been obtained in [10]. The equations of wave propagation in the cubic QCs and the analytical expression of the phase velocity of wave propagation have been derived in [11]. Based on the variation of the general potential function of QCs, the 3D finite element formulation for cubic QCs has been developed in [12].

\footnotetext{
*corresponding author; e-mail: hcerdik@pau.edu.tr
}

Dynamic elasticity problems in 1D, 3D QCs and normal crystals, have been written as a symmetric hyperbolic system of the first order in [13-15]. Applying the Fourier transform to the obtained systems and using some matrix computations, FSs have been computed. In this paper, applying the same procedure to the cubic QCs, phonon-phason displacements, displacement speeds, and stresses arising from pulse point source are computed at the same time. The wave propagation in these crystals is also simulated.

\section{The basic equations for cubic QCs}

According to the generalized elasticity theory for QCs, the generalized Hooke's laws, and dynamic equilibrium equations are given by $[6,12]$ :

$$
\begin{aligned}
& \rho \frac{\partial^{2} u_{i}(x, t)}{\partial t^{2}}=\sum_{j=1}^{3} \frac{\partial \sigma_{i j}(x, t)}{\partial x_{j}}+f_{i}(x, t), \\
& \rho \frac{\partial^{2} w_{i}(x, t)}{\partial t^{2}}=\sum_{j=1}^{3} \frac{\partial H_{i j}(x, t)}{\partial x_{j}}+g_{i}(x, t), \\
& i=1,2,3, \quad x \in R^{3}, \quad t \in R,
\end{aligned}
$$

where the constant $\rho>0$ is the density, $\sigma_{i j}$ and $H_{i j}$ are phonon and phason stresses, $f_{i}(x, t)$ and $g_{i}(x, t)$ are body forces for the phonon and phason displacements, respectively.

The phonon strain $\varepsilon_{k l}$ and phason strain $F_{k l}$ of cubic QCs are given by equations:

$$
\begin{aligned}
\varepsilon_{k l} & =\frac{1}{2}\left(\frac{\partial u_{k}}{\partial x_{l}}+\frac{\partial u_{l}}{\partial x_{k}}\right), \quad F_{k l}=\frac{1}{2}\left(\frac{\partial w_{k}}{\partial x_{l}}+\frac{\partial w_{l}}{\partial x_{k}}\right), \\
k, l & =1,2,3
\end{aligned}
$$

Here $u_{k}$ and $w_{k}$ are phonon and phason displacements, while $\varepsilon_{k l}(x, t)$ and $F_{k l}(x, t)$ are phonon and phason strains, respectively. 
Both equations (1), (2) follow Bak's argument [16, 17]. According to Bak, phasons describe particular types of structural disorder, or structural fluctuations. Furthermore, the phonons and phasons represent wave propagation in real space. The mathematical structure of the theory is relatively simple, and its formulations are similar to that of classical elasto-dynamics. Many researchers followed these formulations to develop the elastodynamics of quasicrystals and make applications in defect dynamics and thermodynamics (for example, see $[1,6,18,19])$.
The phonon stress $\sigma_{i j}$ and phason stress $H_{i j}$ are given in the following vector form [12]:

$$
\boldsymbol{\tau}=C \cdot \boldsymbol{Y} .
$$

Here

$$
\begin{aligned}
\boldsymbol{\tau} & =\left(\sigma_{11}, \sigma_{22}, \sigma_{33}, \sigma_{23}, \sigma_{13}, \sigma_{12}, H_{11}, H_{22}, H_{33},\right. \\
& \left.H_{23}, H_{13}, H_{12}\right)^{*}, \\
\boldsymbol{Y}=\left(\varepsilon_{11}, \varepsilon_{22}, \varepsilon_{33}, 2 \varepsilon_{23}, 2 \varepsilon_{13}, 2 \varepsilon_{12}, F_{11}, F_{22}, F_{33},\right. & \\
& \left.2 F_{23}, 2 F_{31}, 2 F_{12}\right)^{*},
\end{aligned}
$$

$$
C=\left(\begin{array}{cccccccccccc}
c_{1,1} & c_{1,2} & c_{1,2} & 0 & 0 & 0 & R_{1} & R_{2} & R_{2} & 0 & 0 & 0 \\
c_{1,2} & c 1,2 & c_{1,1} & 0 & 0 & 0 & R_{2} & R_{2} & R_{2} & 0 & 0 & 0 \\
c_{1,2} & c 1,2 & c_{1,1} & 0 & 0 & 0 & R_{2} & R_{2} & R_{1} & 0 & 0 & 0 \\
0 & 0 & 0 & c_{4,4} & 0 & 0 & 0 & 0 & 0 & R_{3} & 0 & 0 \\
0 & 0 & 0 & 0 & c_{4,4} & 0 & 0 & 0 & 0 & 0 & R_{3} & 0 \\
0 & 0 & 0 & 0 & 0 & c_{4,4} & 0 & 0 & 0 & 0 & 0 & R_{3} \\
R_{1} & R_{2} & R_{2} & 0 & 0 & 0 & K_{1,1} & K_{1,2} & K_{1,2} & 0 & 0 & 0 \\
R_{2} & R_{1} & R_{2} & 0 & 0 & 0 & K_{1,2} & K_{1,1} & K_{1,2} & 0 & 0 & 0 \\
R_{2} & R_{2} & R_{1} & 0 & 0 & 0 & K_{1,2} & K_{1,2} & K_{1,1} & 0 & 0 & 0 \\
0 & 0 & 0 & R_{3} & 0 & 0 & 0 & 0 & 0 & K_{4,4} & 0 & 0 \\
0 & 0 & 0 & 0 & R_{3} & 0 & 0 & 0 & 0 & 0 & K_{4,4} & 0 \\
0 & 0 & 0 & 0 & 0 & R_{3} & 0 & 0 & 0 & 0 & 0 & K_{4,4}
\end{array}\right)_{12 \times 12}
$$

The symbol $*$ denotes the sign of the transposition, $c_{1,1}$, $c_{1,2}, c_{4,4}$ are the phonon elastic constants, $K_{1,1}, K_{1,2}$, $K_{4,4}$ are the phason elastic constants, and $R_{1}, R_{2}, R_{3}$ are the phonon-phason coupling elastic constants. From the positivity of elastic strain energy density [7], the matrix $C$ is positive definite.

\section{Reduction of equations of anisotropic elastodynamics in cubic QCs to a symmetric hyperbolic system}

Differentiating Eq.(4) with respect to $t$ and multiplying the left hand side of the resulting formula by the inverse of $C$ (denoted as $C^{-1}$ ), we find the following matrix representation

$C^{-1} \frac{\partial \boldsymbol{T}}{\partial t}+\sum_{j=1}^{3}\left(\begin{array}{cc}\left(A_{j}^{1}\right)^{*} & 0_{6,3} \\ 0_{6,3} & \left(A_{j}^{1}\right)^{*}\end{array}\right) \frac{\partial}{\partial x_{j}}\left(\begin{array}{c}\boldsymbol{U} \\ \boldsymbol{W}\end{array}\right)=0_{12,1}$,

where

$$
\begin{aligned}
& A_{1}^{1}=\left(\begin{array}{cccccc}
-1 & 0 & 0 & 0 & 0 & 0 \\
0 & 0 & 0 & 0 & 0 & -1 \\
0 & 0 & 0 & 0 & -1 & 0
\end{array}\right), \\
& A_{2}^{1}=\left(\begin{array}{cccccc}
0 & 0 & 0 & 0 & 0 & -1 \\
0 & -1 & 0 & 0 & 0 & 0 \\
0 & 0 & 0 & -1 & 0 & 0
\end{array}\right),
\end{aligned}
$$

$$
A_{3}^{1}=\left(\begin{array}{cccccc}
0 & 0 & 0 & 0 & -1 & 0 \\
0 & 0 & 0 & -1 & 0 & 0 \\
0 & 0 & -1 & 0 & 0 & 0
\end{array}\right),
$$

$\boldsymbol{U}=\left(U_{1}, U_{2}, U_{3}\right), \quad \boldsymbol{W}=\left(W_{1}, W_{2}, W_{3}\right)$,

$U_{i}(x, t)=\frac{\partial u_{i}(x, t)}{\partial t}, \quad W_{i}(x, t)=\frac{\partial w_{i}(x, t)}{\partial t}, \quad i=1,2,3$.

The $0_{l, n}$ is the zero matrix of the order $l \times n$.

Equations (1), (2) can be written as

$$
\rho \frac{\partial}{\partial t}\left(\begin{array}{c}
\boldsymbol{U} \\
\boldsymbol{W}
\end{array}\right)+\sum_{j=1}^{3}\left(\begin{array}{cc}
A_{j}^{1} & 0_{3,6} \\
0_{3,6} & A_{j}^{1}
\end{array}\right) \frac{\partial \boldsymbol{T}}{\partial x_{j}}=\mathcal{F},
$$

where $\mathcal{F}=\left(f_{1}, f_{2}, f_{3}, g_{1}, g_{2}, g_{3}\right)^{*}$.

The relations (5) and (7) can be represented by

$$
A_{0} \frac{\partial \boldsymbol{V}}{\partial t}+\sum_{j=1}^{3} A_{j} \frac{\partial \boldsymbol{V}}{\partial x_{j}}=\mathbf{F}, \quad x \in R^{3}, \quad t \in R
$$

where

$$
\begin{gathered}
\boldsymbol{F}=\left(f_{1}, f_{2}, f_{3}, g_{1}, g_{2}, g_{3}, 0,0,0,0,0,0,0,0,0,0,0,0\right)^{*}, \\
\boldsymbol{V}=\left(U_{11}, U_{22}, U_{33}, W_{1}, W_{2}, W_{3}, \sigma_{11}, \sigma_{22}, \sigma_{33}, \sigma_{23},\right. \\
\left.\sigma_{13}, \sigma_{12}, H_{11}, H_{22}, H_{33}, H_{23}, H_{13}, H_{12}\right)^{*},
\end{gathered}
$$




$$
\begin{aligned}
A_{0} & =\left(\begin{array}{cc}
\rho_{6} & 0_{6,12} \\
0_{12,6} & C^{-1}
\end{array}\right)_{18 \times 18} \\
A_{j} & =\left(\begin{array}{ccc}
0_{3,6} & A_{j}^{1} & 0_{3,6} \\
0_{3,6} & 0_{3,6} & A_{j}^{1} \\
\left(A_{j}^{1}\right)^{*} & 0_{6,9} & 0_{6,6} \\
0_{6,3} & \left(A_{j}^{1}\right)^{*} & 0_{6,12}
\end{array}\right)_{18 \times 18}
\end{aligned}
$$

We note that the matrices $A_{j}$, for $j=1,2,3$, are symmetric. Since $C$ is positive definite and symmetric, and $\rho>0$, the matrix $A_{0}$ is symmetric and positive definite. Therefore, system (8) is a symmetric hyperbolic system [20].

\section{Fundamental solution of anisotropic elastodynamics in cubic QCs}

In this section, we give explicit formula of the FS for the equations of elastodynamics in $3 \mathrm{D}$ cubic QC.

Let $m$ run values $1,2,3,4,5,6$. The time-dependent FS of elasticity for cubic QCs is a $18 \times 6$ matrix, whose $m$-th column is a vector function

$$
\begin{gathered}
\boldsymbol{V}^{m}(x, t)=\left(U_{1}^{m}(x, t), U_{2}^{m}(x, t), U_{3}^{m}(x, t), W_{1}^{m}(x, t),\right. \\
W_{2}^{m}(x, t), W_{3}^{m}(x, t), \sigma_{11}^{m}(x, t), \sigma_{22}^{m}(x, t), \sigma_{33}^{m}(x, t), \\
\sigma_{23}^{m}(x, t), \sigma_{13}^{m}(x, t), \sigma_{12}^{m}(x, t), H_{11}^{m}(x, t), H_{22}^{m}(x, t), \\
\left.H_{33}^{m}(x, t), H_{23}^{m}(x, t), H_{31}^{m}(x, t), H_{12}^{m}(x, t)\right)^{*}
\end{gathered}
$$

satisfying the following IVP

$A_{0} \frac{\partial \boldsymbol{V}^{m}}{\partial t}+\sum_{j=1}^{3} A_{j} \frac{\partial \mathbf{V}^{m}}{\partial x_{j}}=\boldsymbol{E}^{m} \delta(x, t), \quad x \in R^{3}, \quad t \in R$

$$
\left.\boldsymbol{V}^{m}(x, t)\right|_{t<0}=0 \text {. }
$$

Here, $\boldsymbol{E}^{m}=\left(\delta_{1}^{m}, \delta_{2}^{m}, \delta_{3}^{m}, \delta_{4}^{m}, \delta_{5}^{m}, \delta_{6}^{m}, 0,0,0,0,0,0,0,0\right.$, $0,0,0,0)^{*}, \delta_{n}^{m}$ denotes the Kronecker symbol, i.e., $\delta_{n}^{m}=1$ if $n=m$, and $\delta_{n}^{m}=0$ if $n \neq m$, for $n, m=1,2,3,4,5,6$. The $\delta(x)=\delta\left(x_{1}\right) \delta\left(x_{2}\right) \delta\left(x_{3}\right)$ is the Dirac delta function of the space variable concentrated at $x_{1}=0, x_{2}=0$, $x_{3}=0$. The $\delta(t)$ is the Dirac delta function of the time variable concentrated at $t=0$. Applying the Fourier transformation to the IVP (10) and (11) with respect to $x \in R^{3}$ and using the matrix transformations, an explicit formula for $m$-th column of the FS is found [13-15] by the inverse Fourier transform as follows:

$$
\begin{aligned}
& \boldsymbol{V}^{m}(x, t)=\frac{\theta(t)}{(2 \pi)^{3}} \int_{-\infty}^{\infty} \int_{-\infty}^{\infty} \int_{-\infty}^{\infty} \mathcal{T}(\nu) \cos (D(\nu) t-\boldsymbol{I}(\nu \cdot x)) \\
& \quad \times \mathcal{T}^{*}(\nu) \boldsymbol{E}^{m} \mathrm{~d} \nu_{1} \mathrm{~d} \nu_{2} \mathrm{~d} \nu_{3},
\end{aligned}
$$$$
\boldsymbol{V}^{m}(x, t)=\left(V_{1}(x, t), V_{2}(x, t), V_{3}(x, t), \ldots, V_{18}(x, t)\right)^{*},
$$

where $\cos (D(\nu) t-\boldsymbol{I}(\nu \cdot x))$ is the diagonal matrix. Non-singular matrix $\mathcal{T}(\nu)$ and a diagonal matrix $D(\nu)=\operatorname{diag}\left(d_{k}(\nu)\right)$, for $k=1,2, \ldots, 18$, with real valued elements, can be computed as follows

$$
\begin{aligned}
& \mathcal{T}^{*}(\nu) A_{0} \mathcal{T}(\nu)=I, \\
& \mathcal{T}^{*}(\nu)\left(\nu_{1} A_{1}+\nu_{2} A_{2}+\nu_{3} A_{3}\right) \mathcal{T}(\nu)=D(\nu),
\end{aligned}
$$

where $I$ is the identity matrix, $\mathcal{T}^{*}(\nu)$ is the transposed matrix to $\mathcal{T}(\nu)$.

Integrating the first six components of $\boldsymbol{V}^{m}(x, t)$ with respect to $t$, the FS for phonon and phason displacements of elastodynamics of cubic QCs can be found [13-15] as follows

$$
\begin{aligned}
& u_{n}^{m}(x, t)=\frac{\theta(t)}{(2 \pi)^{3}} \\
& \times \int_{-\infty}^{\infty} \int_{-\infty}^{\infty} \int_{-\infty}^{\infty}\left[\mathcal{T}(\nu) S(\nu, t, x) \mathcal{T}^{*}(\nu) \boldsymbol{E}^{m}\right]_{n} \mathrm{~d} \nu_{1} \mathrm{~d} \nu_{2} \mathrm{~d} \nu_{3} \\
& w_{n}^{m}(x, t)=\frac{\theta(t)}{(2 \pi)^{3}} \\
& \times \int_{-\infty}^{\infty} \int_{-\infty}^{\infty} \int_{-\infty}^{\infty}\left[\mathcal{T}(\nu) S(\nu, t, x) \mathcal{T}^{*}(\nu) \boldsymbol{E}^{m}\right]_{n+3} \mathrm{~d} \nu_{1} \mathrm{~d} \nu_{2} \mathrm{~d} \nu_{3},
\end{aligned}
$$

where $n=1,2,3$, and components of the matrix $S$ are given by

$$
\begin{aligned}
& S_{k k}(\nu, t)= \begin{cases}\frac{\sin \left(d_{k}(\nu) t-\nu x\right)}{d_{k}(\nu)}+\frac{\sin (\nu x)}{d_{k}(\nu)}, & \text { if } \quad d_{k}(\nu) \neq 0, \\
t \cos (\nu x), & \text { if } d_{k}(\nu)=0,\end{cases} \\
& S_{k j}(\nu, t, x)=0, \quad j \neq k, \quad k, j=1, \ldots, 18 .
\end{aligned}
$$

\section{Application}

In this section, we compute and simulate the FS of the elastodynamics in 3D cubic QC.

Since elastic constants for cubic QCs are not available presently, we choose $\rho=1 \times 10^{3}\left[\mathrm{~kg} / \mathrm{m}^{3}\right]$ and

$c_{1,1}=112.1, \quad c_{1,2}=60.3, \quad c_{4,4}=32.8$,

$R_{1}=0.5, \quad R_{2}=-0.2, \quad R_{4}=0.7$,

$K_{1,1}=300, \quad K_{1,2}=100, \quad K_{4.4}=50 \times\left[10^{10} \mathrm{~Pa}\right] . \quad(19)$ Using the presented method in Sect. 4 , we compute the solution

$$
\boldsymbol{V}^{2}(x, t)=\left(V_{1}^{2}(x, t), V_{2}^{2}(x, t), V_{3}^{2}(x, t), \ldots, V_{18}^{2}(x, t)\right)
$$

of problem (10) and (11). We give the simulations of the computational experiment in Figs. 1-10.

Figures 1 and 2 present 2D level plots of the second and third phason displacements $w_{2}^{2}\left(0, x_{2}, x_{3}, 0.1\right)$ and $w_{3}^{2}\left(0, x_{2}, x_{3}, 0.1\right)$, respectively. These figures present view from the top of the magnitude axes $w_{2}^{2}\left(0, x_{2}, x_{3}, 0.1\right)$ and $w_{3}^{2}\left(0, x_{2}, x_{3}, 0.1\right)$. Figures $3-5$ show simulation of the second phonon speed $U_{2}^{2}\left(0, x_{2}, x_{3}, t\right)$ for different times $t=$ $0.05,0.1,0.25$. Figures 4 , and 5 show $3 \mathrm{D}$ and $2 \mathrm{D}$ plots of dynamic distribution for the second component of the phonon stress $\sigma_{22}^{2}\left(0, x_{2}, x_{3}, 0.1\right)$. Figure 4 shows $3 \mathrm{D}$ plot of the $\sigma_{22}^{2}\left(0, x_{2}, x_{3}, t\right)$ at the time $t=$ 0.1. Here, the horizontal axes are $x_{2}$ and $x_{3}$. 


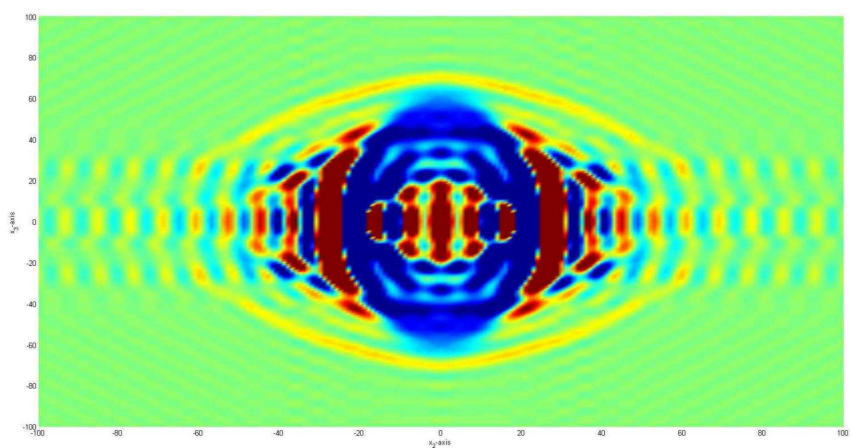

Fig. 1. The second component of the phason displacement $w_{2}^{2}\left(0, x_{2}, x_{3}, t\right)$ at time $t=0.1$.

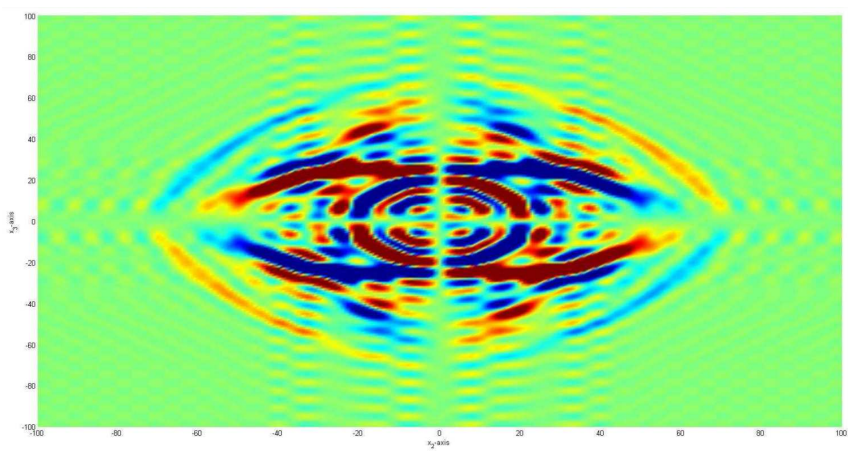

Fig. 2. The third component of the phason displacement $w_{3}^{2}\left(0, x_{2}, x_{3}, t\right)$ at time $t=0.1$.

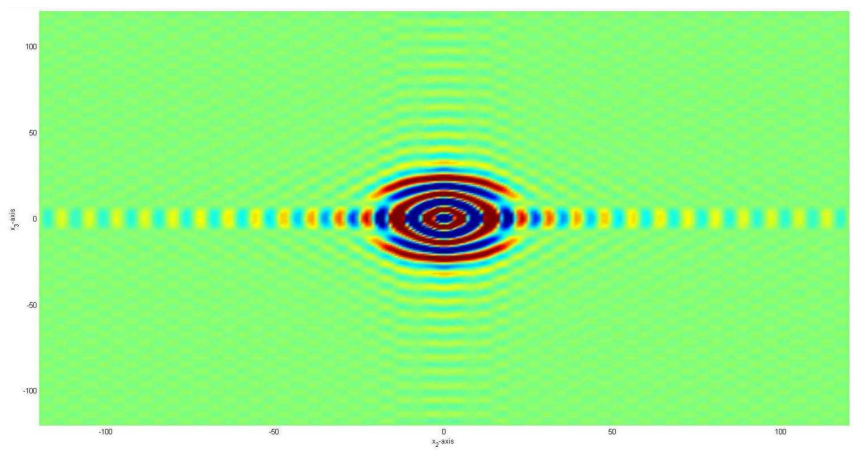

Fig. 3. The second component of the phonon displacement speed $U_{2}^{2}\left(0, x_{2}, x_{3}, t\right)$ at time $t=0.05$.

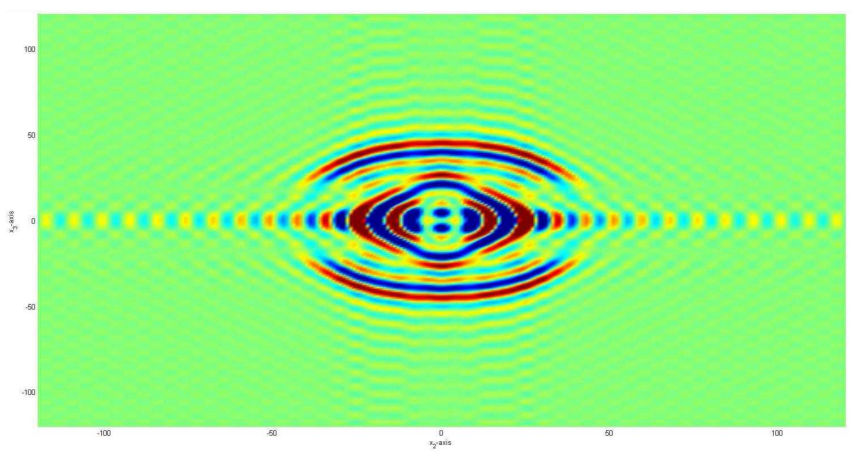

Fig. 4. The second component of the phonon displacement speed $U_{2}^{2}\left(0, x_{2}, x_{3}, t\right)$ at time $t=0.1$.

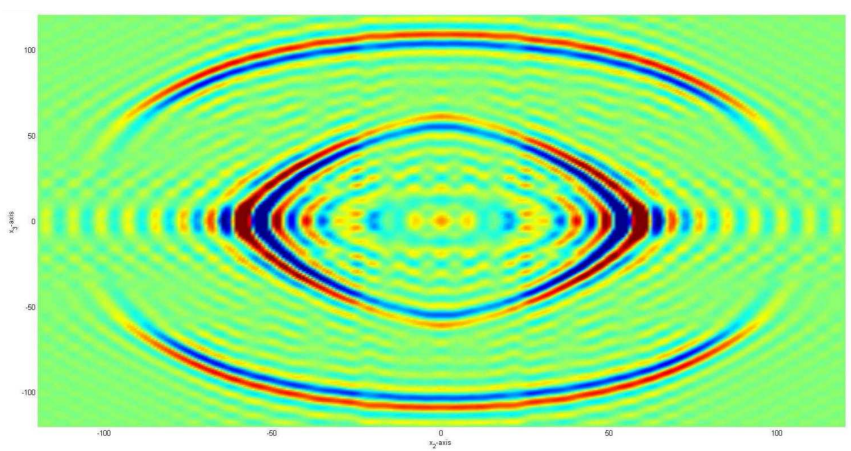

Fig. 5. The second component of the phonon displacement speed $U_{2}^{2}\left(0, x_{2}, x_{3}, t\right)$ at time $t=0.25$.

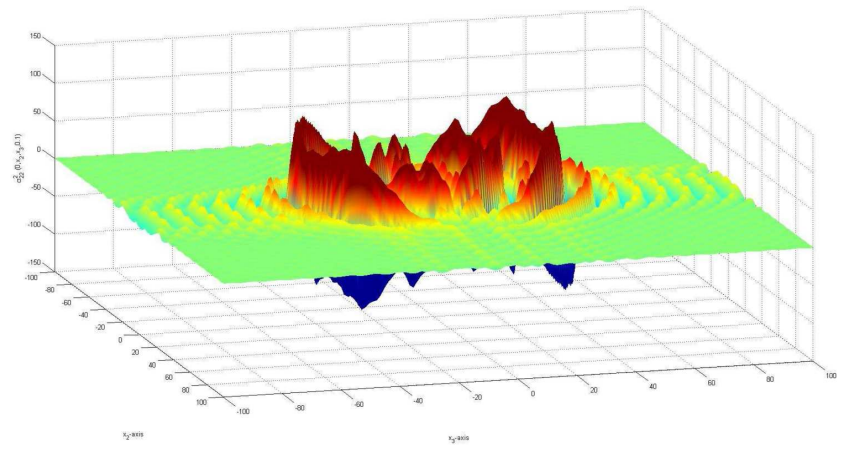

Fig. 6. The second component of the phonon stress $\sigma_{22}^{2}\left(0, x_{2}, x_{3}, t\right)$ at time $t=0.1$.

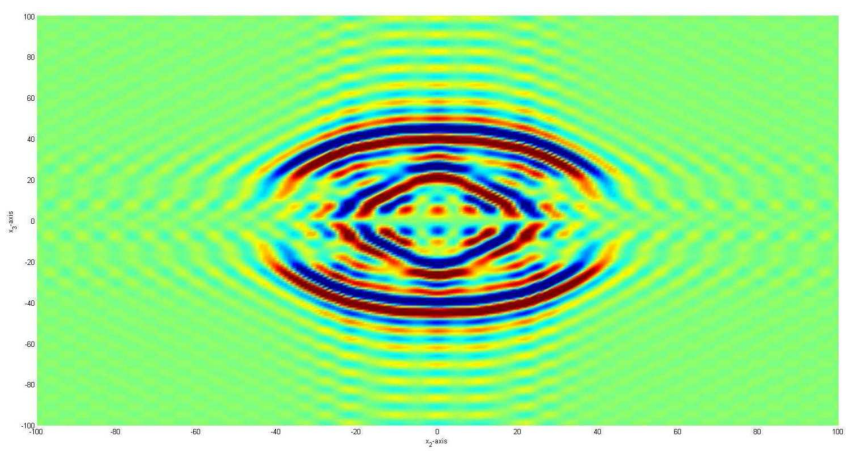

Fig. 7. The second component of the phonon stress $\sigma_{22}^{2}\left(0, x_{2}, x_{3}, t\right)$ at time $t=0.1$.

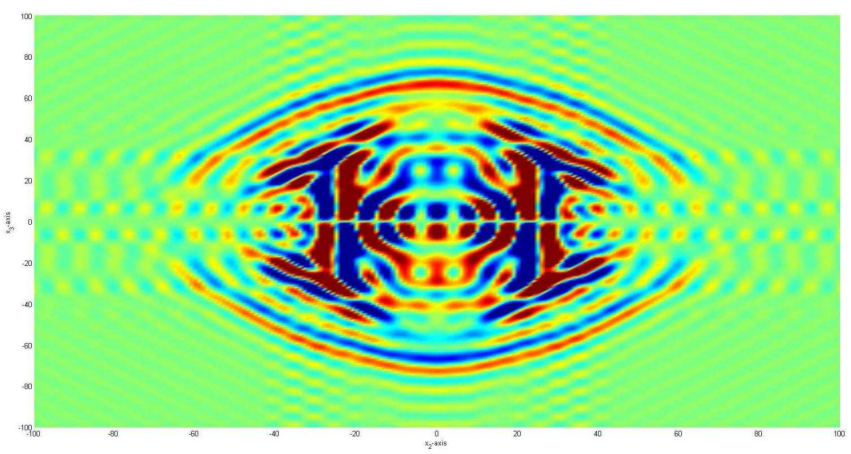

Fig. 8. The second component of the phason stress $H_{22}^{2}\left(0, x_{2}, x_{3}, t\right)$ at time $t=0.1$. 


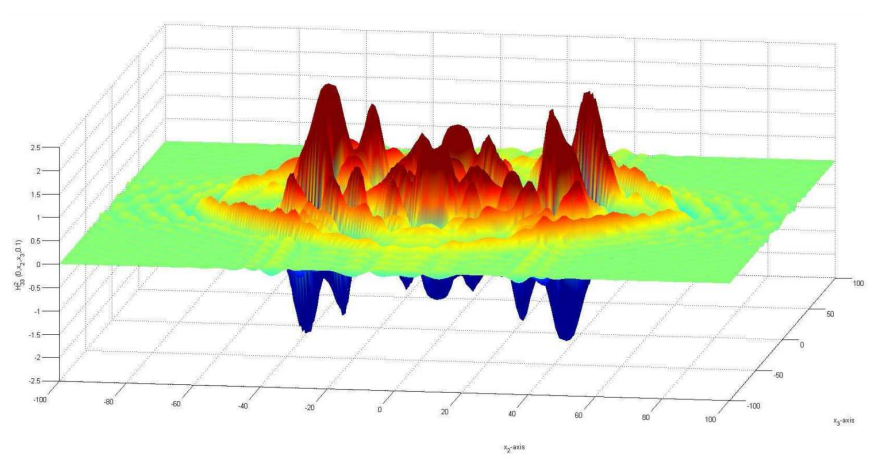

Fig. 9. The third component of the phason stress $H_{33}^{2}\left(0, x_{2}, x_{3}, t\right)$ at time $t=0.1$.

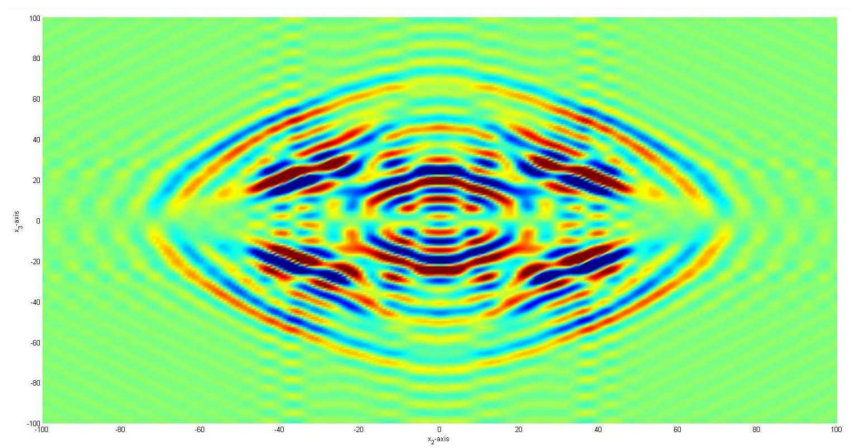

Fig. 10. The third component of the phason stress $H_{33}^{2}\left(0, x_{2}, x_{3}, t\right)$ at time $t=0.1$.

The vertical axis is the magnitude of $\sigma_{22}^{2}\left(0, x_{2}, x_{3}, 0.1\right)$. Figure 5 shows 2D plot of dynamic distribution for the second component of the phonon stress $\sigma_{22}^{2}\left(0, x_{2}, x_{3}, 0.1\right)$. This figure presents view from the top of the magnitude axis $\sigma_{22}^{2}\left(0, x_{2}, x_{3}, 0.1\right)$. Figure 6 shows 2D plot of dynamic distribution for the second component of the phason stress $H_{22}^{2}\left(0, x_{2}, x_{3}, 0.1\right)$. Figures 7 and 8 show 3D and 2D plots of dynamic distribution for the third component of the phason stress $H_{33}^{2}\left(0, x_{2}, x_{3}, 0.1\right)$.

In this example, the wave propagation of the second and third phason displacements, the second phonon displacement speed, the second phonon and the third phason stresses in 3D cubic QCs arising from pulse point sources $\mathbf{E}^{2} \delta(x, t)$ have been given. Since our problem follows Bak's argument, the solutions for the phonon and phason fields are dominated by wave propagation.

\section{Conclusion}

To our knowledge, in the literature, simulation of the elastic wave propagation arising from pulse point sources in 3D cubic QCs has not yet been obtained.
In this paper, FS for phonon and phason displacements, displacement speeds, and stresses in 3D cubic QCs has been computed by using Fourier transformation and some matrix computations. As an application, simulations of the FSs of the phonon and phason displacements, displacement speeds, and stresses for 3D cubic QC have been given by using MATLAB programming. The results of simulations provide with a possibility to observe and analyze the elastic wave propagation arising from pulse point sources in 3D cubic QCs.

\section{References}

[1] T.Y. Fan, Mathematical Theory of Elasticity of Quasicrystals and Its Applications, Springer-Verlag, Berlin 2011.

[2] Y.C. Feng, G.H. Lu, R.L. Withers, J. Phys. Condens. Matter 1, 3695 (1989).

[3] Y.C. Feng, D.S. Zhou, D.X. Li, C. Dong, G.V. Tendeloo, K.H. Kuo, Philos Mag. Lett. 55, 221 (1987).

[4] Y.C. Feng, G. Lu, H.Q. Ye, K.H. Ruo, R.L. Withers, G.V. Tendeloo, J. Phys. Condens. Matter 2, 9749 (1990).

[5] R.H. Wang, C.G. Qin, G.H. Lu, Y.C. Feng, S.Q. Xu, Acta Cryst. A 50, 366 (1994).

[6] W.G. Yang, R.H. Wang, D.H. Ding, C.Z. Hu, Phys. Rev. B 48, 6999 (1993).

[7] Y. Gao, A. Ricoeur, L.L. Zhang, Phys. Lett. A 375, 2775 (2011)

[8] Y. Gao, Phys. Lett. A 373, 885 (2009).

[9] Y. Gao, A. Ricoeur, Phys. Lett. A 374, 4354 (2010).

[10] W. Zhou, T. Fan, Chin. Phys. 9, 294 (2000).

[11] C. Li, Y. Liu, W. Sritrakool, Scienceasia 27, 67 (2001).

[12] L.Z. Yang, F.M. He, Y. Gao, Acta Phys. Pol. A 126, 471 (2014).

[13] H.C. Yaslan, App. Math. Modell. 37, 8409 (2013).

[14] H.C. Yaslan, Wave Random Complex 29, 614 (2019).

[15] V.G. Yakhno, H.C. Yaslan, Comput. Struct. 89, 646 (2011).

[16] P. Bak, Phys. Rev. Lett. 54, 1517 (1985).

[17] P. Bak, Phys. Rev. B 32, 5764 (1985).

[18] W. Shi, Eur. J. Mech. A Solids 24, 217 (2005).

[19] T.Y. Fan and Y.W. Mai, Appl. Mech. Rev. 57, 325 (2004).

[20] P.T. Lax, Hyperbolic Partial Differential Equations, American Mathematical Society, Providence (RI) 2006. 